\title{
New Problems - New Solutions: A Never Ending Story
}

\section{Findings from the Clinical Information Systems Perspective for 2015}

\author{
W.O. Hackl ${ }^{*}$, T. Ganslandt ${ }^{2 *}$, Section Editors for the IMIA Yearbook Section on Clinical \\ Information Systems \\ 1 Institute of Biomedical Informatics, UMIT-University of Health Sciences, Medical Informatics and \\ Technology, Hall in Tirol, Austria \\ 2 Medical Center for Information and Communication, Erlangen University Hospital, Erlangen, Germany \\ * Equal Contribution
}

\begin{abstract}
Summary
Objective: To summarize recent research and to propose a selection of best papers published in 2015 in the field of Clinical Information Systems (CIS).

Method: The query which had been used last year to retrieve articles for the CIS section of the IMIA Yearbook of Medical Informatics 2015 was refined. It again aimed at identifying relevant publications in the field of CIS and comprised search terms from the Medical Subject Headings (MeSH) catalog as well as additional free text search terms from PubMed and Web of Science. The retrieved articles were categorized in a multi-pass review carried out separately by the two section editors. The final selection of 15 candidate papers was then peer-reviewed by Yearbook editors and external reviewers. Based on the review results the four best papers were then selected at the best papers selection meeting with the IMIA Yearbook editorial board. To get an overview on the content of the retrieved articles we applied text mining and term co-occurrence mapping techniques. Results: The query was carried out in mid-January 2016, yielding a combined result set of 1851 articles which were published in 790 different journals. The most relevant terms from abstracts and titles of these articles were assigned to six different clusters. A majority of articles dealt with two thematic blocks, problems and solutions in the CIS field. The majority of the 2016 CIS candidate papers and all four best papers could be assigned to these two thematic blocks. Conclusions: We identified two main tracks among the CIS candidate and best papers as well as in CIS research activities in general: problems and solutions. A never ending cycle of continuous improvement.
\end{abstract}

\section{Keywords}

Medical informatics, International Medical Informatics Association, yearbook, clinical information systems

Yearb Med Inform 2016:146-51 http://dx.doi.org/10.15265/IY-2016-054

Published online November 10, 2016

\section{Introduction}

In the last year's synopsis for the Clinical Information Systems (CIS) section of the IMIA Yearbook of Medical Informatics [1] we concluded that CIS had become mature over the last years and that the focus of recent research in the CIS field had moved beyond simple data acquisition for just supporting local care workflows. We found out that - to a great extent - current research efforts comprised the breakdown of information silos, the reduction of barriers between different systems and secondary use of accumulated health data for multiple purposes.

We also noticed that the CIS domain is closely intertwined with the domains of other Yearbook sections, especially Clinical Decision Support Systems (CDSS), Clinical Research Informatics (CRI) and Human Factors (HF), as we discovered significant overlap between the result sets of our and those section editors' queries. Therefore we tried to sharpen our query to minimize such overlaps. And it seems that our efforts were successful. Although the number of retrieved papers increased by nearly 20 percent compared to the previous year, the result set seemed to be more specific and containing a higher density of CIS papers.

\section{About the Paper Selection}

The selection process for the CIS section again followed the process described in [2]. Relevant search terms were again retrieved first from the Medical Subject Headings
(MeSH) catalog, covering major topics from hospital records, health information exchange, health and clinical information systems and meaningful use, among others. Then, additional free text search terms covering the same topics retrieved from $\mathrm{MeSH}$ were derived and searched within the title and abstract fields of PubMed, as indexing with MeSH keywords may occur with a lag of several months after submission to PubMed. As in the previous year, free-text search was also restricted to citations labeled with status "publisher" or "inprocess", which signifies the lack of MeSH-coding.

The query was further restricted to journal articles published in 2015 in English language and including an abstract. Although classified as journal articles by PubMed, all conference papers published in Studies in Health Technology and Informatics were also excluded from the final result set. Topics from other IMIA Yearbook sections were excluded with appropriate $\mathrm{MeSH}$ and free text terms. Articles present in the 2015 IMIA Yearbook were likewise excluded. Additional exclusions by publication type were added to restrict the query to original research articles and reviews. The free-text query was additionally reformatted for Web of Science ${ }^{\circledR}$ (from Thomson Reuters) with an additional restriction to the subject area "Medical Informatics". The full queries are available upon request from the corresponding authors.

The queries were carried out in mid-January 2016, yielding a combined result set from PubMed and Web of Science of 1851 articles. The resulting articles were published 
in 790 different journals. Figure 1 depicts the top ten journals with the highest numbers of resulting articles.

The query results were loaded into the BibReview software [2] for a multi-pass review carried out separately by the two section editors (TG, WOH), with first-pass selection based on titles and selection based on abstracts at second-pass, and also based on full-texts henceforward as required. Results were merged, and articles tagged with pending or conflicting status jointly re-assessed in a total of five passes, yielding a final selection of 15 candidate papers. These papers were then peer-reviewed by Yearbook editors and external reviewers. Four papers were selected as best papers for the CIS section (Table 1). A content summary of the 4 selected papers can be found in the appendix of this synopsis.

\section{Findings and Trends: Clinical Information Systems 2015}

Last year we analyzed key word frequencies to gain an overview on the content of all retrieved articles. This year we used a more sophisticated text mining approach to gain more insights and created a co-occurrence map of most relevant terms derived from the titles and abstracts of the articles in our CIS result set. To calculate and visualize this map we used VOSviewer, a software tool for constructing and visualizing bibliometric networks [3]. More details concerning the clustering and map layouting techniques can be found in [4] .

Figure 2 depicts the resulting co-occurrence map of the most relevant terms (top-60 percent, $n=344$ ).

Six different clusters of terms could be identified. The largest cluster in red on bottom right contains 129 terms and can be seen as "core cluster" of the CIS domain dealing with clinical/health information systems and electronic medical/patient/health/ personal records in all forms and flavors as well as other health-IT applications, their functionalities, requirements and challenges, including meaningful use and health information exchange amongst others.

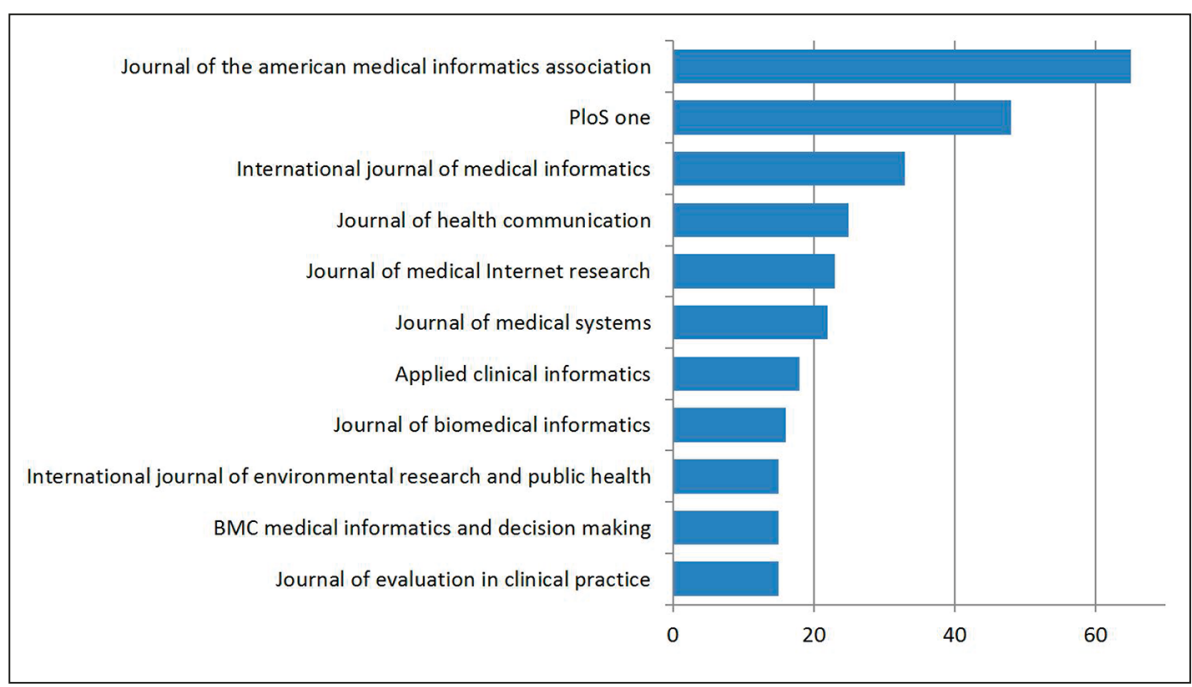

Fig. 1 Number of retrieved articles for Top-10 journals

Table 1 Best paper selection of articles for the IMIA Yearbook of Medical Informatics 2016 in the section 'Clinical Information Systems'. The articles are listed in alphabetical order of the first author's surname.

Section

Clinical Information Systems

- Pickering BW, Dong Y, Ahmed A, Giri J, Kilickaya O, Gupta A, Gajic 0, Herasevich V. The implementation of clinician designed, human-centered electronic medical record viewer in the intensive care unit: a pilot step-wedge cluster randomized trial. Int J Med Inform 2015 May;84(5):299-307.

- Slight SP, Eguale T, Amato MG, Seger AC, Whitney DL, Bates DW, Schiff GD. The vulnerabilities of computerized physician order entry systems: a qualitative study. J Am Med Inform Assoc 2016 Mar;23(2):311-6.

- Varpio L, Rashotte J, Day K, King J, Kuziemsky C, Parush A. The EHR and building the patient's story: A qualitative investigation of how EHR use obstructs a vital clinical activity. Int J Med Inform 2015 Dec;84(12): 1019-28.

- Wright A, McCoy AB, Hickman TT, Hilaire DS, Borbolla D, Bowes WA 3rd, Dixon WG, Dorr DA, Krall M, Malholtra S, Bates DW, Sittig DF. Problem list completeness in electronic health records: A multi-site study and assessment of success factors. Int J Med Inform 2015 0ct;84(10):784-90.

The second largest cluster depicted in green on the bottom left contains 100 terms that can be related to the context of the articles. Mainly terms related to study types or designs (e.g. trial, cohort study, retrospective study), to study population, inclusion or exclusion criteria (e.g. age group, sex, disease), to study objectives as well as to outcomes or other relevant context factors (incidence, mortality, cause, exposure, risk, rates, survival, etc.) were assigned to this cluster.

The third largest cluster on top $(\mathrm{n}=59)$, colored in blue primarily contains location related terms (e.g. geographic information system, region, study area, distance, spatial distribution).
Two of the remaining three small clusters deal with other study characteristics or settings (yellow $n=25$, mint green $n=12$ ). The pink cluster $(\mathrm{n}=19)$ contains terms related to adverse events, their detection, reporting and prevention.

These findings are congruent with the results of the key word analysis which was performed last year, although we feel that this year the query was more precise and the results were more specific for the CIS section.

When we assessed the 1851 articles we again found a striking proportion of articles dealing with interoperability from different points of view and with secondary use of existing health data in all its shades as in the 
Hackl et al.

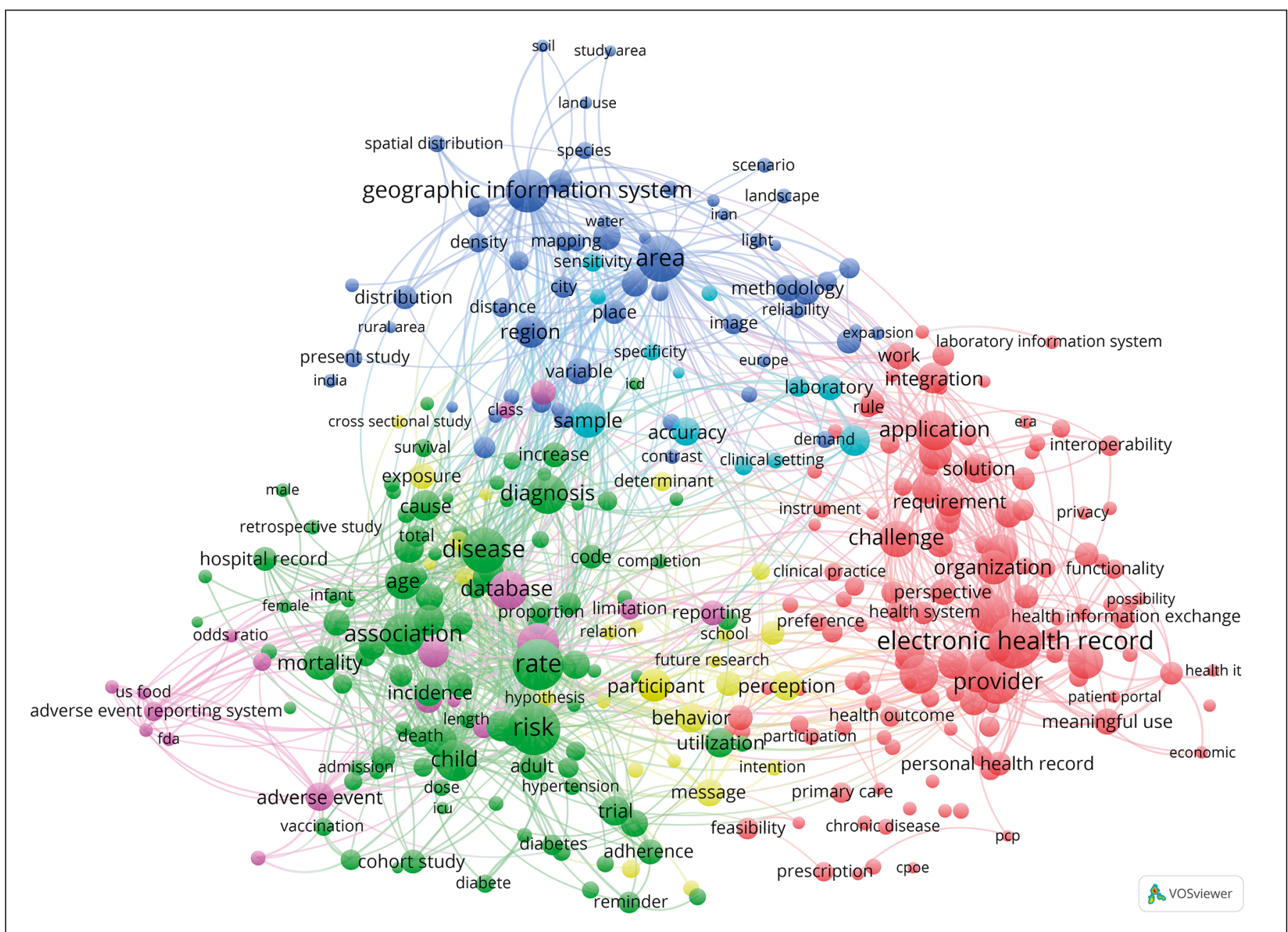

Fig. 2 Clustered co-occurrence map of the most relevant terms (top 60 percent, $n=344$ ) from titles and abstracts of the 1851 papers in the 2016 CIS query result set. Only terms that were found in at least ten different papers were included in the analysis. Node size corresponds to the frequency of the terms (binary count, once per paper). Edges indicate co-occurrence and distance of nodes corresponds to the association strength of the terms within the texts. Colors represent different clusters.

last year. Then, we identified a trend towards publications reporting from benefits as well as risks due to CIS. Now, we found that this trend continued as we found many papers dealing with problems and proposing solutions in the CIS field among the CIS result set. It is remarkable that the majority of the 2016 CIS candidate papers and all four best papers can be assigned to these two thematic blocks, problems and solutions.

One of our 2016 CIS section best papers in the problems block comes from Lara Varpio and colleagues [5]. Their well-designed qualitative study involved multiple data sources and revealed "that EHR use obstructed clinicians' ability to build the patient's story by fragmenting data interconnections". Their results can guide the way implementation should be done to support clinical reasoning and communication. The next best paper in the problems block comes from Sarah Slight and colleagues [6]. Their very interesting article presents an evaluation of 13 commercially available or locally developed CPOE systems and reminds us once again that "CPOE systems often fail to detect and prevent important medication errors" or that they even tend to produce new problems and errors. We also had one candidate paper from Chia-Chen Hsu and colleagues in the problems block. Their novel and important study calls for better contingency when $\mathrm{CPOE}$ and other health IT interventions experience periods of unavailability and calls our attention to the danger of technology dependence [7].

Fortunately, CIS do not only cause problems. In fact they can be very useful, can provide new solutions for existing problems and can bring manifold benefits. Ten of our 2016 CIS candidate papers including the two remaining best papers are representative for a multitude of articles describing such positive effects of CIS. The solutions block can be further divided in four subsections. The first deals with organizational factors and contains the next 2016 CIS best paper. 
It is from Adam Wright and colleagues who identified several success factors associated with problem list completeness [8]. Dean Sittig and colleagues presented a compact compilation of patient safety goals in their perspective paper on the proposed Federal Health Information Technology Safety Center [9]. Therefore we selected it as a candidate paper.

The next subsection in the solutions block deals with improved information presentation. Here we find the final best paper in the 2016 CIS section. Brian Pickering and colleagues report from the implementation of a novel EMR dashboard which had been designed by clinicians in four ICU sites at Mayo Clinic to support bedside clinical information management [10]. Although generalization of results outside of the Mayo environment can be seen as a limitation, we can learn a lot about how such a tool should be designed. Three other candidate papers also deal with improved information presentation. A study from Susanna Shaw and colleagues investigated the effect of a real-time safety bundle dashboard on quality improvement measures in a pediatric ICU environment [11]. David Cook and colleagues report from the development and evaluation of a comprehensive, EMR integrated information management system for bedside teaching and learning support [12]. It should be noted that this is also a relevant example of secondary use of existing data. Besides using existing data, it will be necessary to have a look at possibilities how to deal with new data sources being available at the bedside in the future. In their candidate paper, Jeremy Warner and colleagues propose a concept and pilot for a software package for networked, interactive phenotype visualization for clinician use [13].

The following two candidate papers lead us to the next subsection in the solutions block: leveraging EHR data. Allison McCoy and colleagues validated a crowdsourcing methodology for generating a knowledge base of problem-medication pairs and obtained quite promising results with their approach [14]. Jens Meier and colleagues used stochastic models based on Hidden Markov Models to predict clinical workflow steps from patient specific information; an interesting approach which may be beneficial for the development of clinical workflow assistance functionalities [15].

The last subsection in the solutions block is dedicated to papers providing solutions for measuring benefits. Brian Dekarske and colleagues present a simple but effective solution for an old but still omnipresent problem, alert overriding. In a cross-over RCT they successfully demonstrated the benefits of using a customized list of override reasons in a CPOE system on the appropriateness of override reasons [16]. The other, also very worth reading candidate paper in this subsection comes from Jessica Ancker and colleagues who investigated the relationship between physician use of individual EHR functions and healthcare quality [17].

Among the 15 candidate papers only two could not be assigned to this year's main blocks problems and solutions. They have to be assigned to a third block which is also important in the CIS context: managing complex interoperability. One candidate paper in this block is from Sebastian Stäubert and colleagues who describe a meta-modelling approach and a tool to help document health IT standards, specifically IHE profiles [18]. The other candidate paper from Sara Marceglia and colleagues focuses on one of the major limitations that are underlying the long-term effectiveness of mHealth Apps and proposes a standards-based architecture for integrating such apps to EHR systems [19].

In addition to the candidate papers and the selected best papers we want to highlight an additional paper at this point, our first "just not" candidate paper (rank 16), nevertheless a very noteworthy contribution from Bulent Oral and colleagues [20]. The paper highlights an important issue in health information management and clinical decision support associated with the use of timely laboratory data and offers an excellent model for a solution for Laboratory Information System downtimes. In addition the paper illustrates that even very short and infrequent downtimes of crucial CIS components can have dramatic effect on clinical activities. It also reminds us to keep track of the increasing relevance of CIS emergency concepts for IT-blackouts or disasters. In this spirit we want to recommend a reading of this year's survey article in the CIS section [21].

\section{Conclusions and Outlook}

We identified two main tracks among the CIS candidate and best papers as well as in CIS research activities in general: problems and solutions. We detected a variety of publications reporting from problems related to CIS or inflicted by CIS themselves. On the other hand we identified a majority of publications reporting from successful implementations, working solutions and various benefits caused by CIS. The nature of things, of course is also true for CIS: problem and solution, new problem and new solution. Continuous improvement...

\section{Acknowledgements}

We would like to acknowledge the support of Martina Hutter, Chris Lehmann, MarieChristine Jaulent, Brigitte Seroussi, Ross Koppel and the whole Yearbook editorial team as well as the numerous reviewers in the selection process of the best papers.

\section{References}

1. Ganslandt T, Hackl WO. Findings from the Clinical Information Systems Perspective. Yearb Med Inform 2015 Aug 13;10(1):90-4.

2. Lamy JB, Seroussi B, Griffon N, Kerdelhue G, Jaulent MC, Bouaud J. Toward a formalization of the process to select IMIA Yearbook best papers. Methods Inf Med 2015;54(2):135-44.

3. van Eck NJ, Waltman L. Software survey: VOSviewer, a computer program for bibliometric mapping. Scientometrics 2010 Aug;84(2):523-38.

4. Waltman L, van Eck NJ, Noyons ECM. A unified approach to mapping and clustering of bibliometric networks. JOI Journal of Informetrics 2010;4(4):629-35.

5. Varpio L, Rashotte J, Day K, King J, Kuziemsky $\mathrm{C}$, Parush A. The EHR and building the patient's story: A qualitative investigation of how EHR use obstructs a vital clinical activity. Int J Med Inform 2015 Dec;84(12):1019-28.

6. Slight SP, Eguale T, Amato MG, Seger AC, Whitney DL, Bates DW, Schiff GD. The vulnerabilities of computerized physician order entry systems: a qualitative study. J Am Med Inform Assoc 2015 Mar;23(2):311-6.

7. Hsu CC, Chou CL, Chen TJ, Ho CC, Lee CY, Chou YC. Physicians Failed to Write Flawless Prescriptions When Computerized Physician Order Entry System Crashed. Clin Ther 2015 May 1;37(5):1076-80 e1.

8. Wright A, McCoy AB, Hickman TT, Hilaire DS, Borbolla D, Bowes WA, 3rd, Dixon WG, Dorr 
DA, Krall M, Malholtra S, Bates DW, Sittig DF. Problem list completeness in electronic health records: A multi-site study and assessment of success factors. Int J Med Inform 2015 Oct;84(10):784-90.

9. Sittig DF, Classen DC, Singh H. Patient safety goals for the proposed Federal Health Information Technology Safety Center. J Am Med Inform Assoc 2015 Mar;22(2):472-8.

10. Pickering BW, Dong Y, Ahmed A, Giri J, Kilickaya O, Gupta A, Gajic O, Herasevich V. The implementation of clinician designed, human-centered electronic medical record viewer in the intensive care unit: a pilot step-wedge cluster randomized trial. Int J Med Inform 2015 May;84(5):299-307.

11. Shaw SJ, Jacobs B, Stockwell DC, Futterman C, Spaeder MC. Effect of a Real-Time Pediatric ICU Safety Bundle Dashboard on Quality Improvement Measures. Jt Comm J Qual Patient Saf 2015 Sep;41(9):414-20.

12. Cook DA, Sorensen KJ, Nishimura RA, Ommen SR, Lloyd FJ. A comprehensive information technology system to support physician learning at the point of care. Acad Med 2015 Jan;90(1):33-9.

13. Warner JL, Denny JC, Kreda DA, Alterovitz G. Seeing the forest through the trees: uncovering phenomic complexity through interactive network visualization. J Am Med Inform Assoc 2015 Mar;22(2):324-9.

14. McCoy AB, Wright A, Krousel-Wood M, Thomas EJ, McCoy JA, Sittig DF. Validation of a Crowdsourcing Methodology for Developing a Knowledge Base of Related Problem-Medication Pairs. Appl Clin Inform 2015;6(2):334-44.

15. Meier J, Dietz A, Boehm A, Neumuth T. Predicting treatment process steps from events. J Biomed Inform 2015 Feb;53:308-19.

16. Dekarske BM, Zimmerman CR, Chang R, Grant PJ, Chaffee BW. Increased appropriateness of customized alert acknowledgement reasons for overridden medication alerts in a computerized provider order entry system. Int J Med Inform 2015 Dec;84(12):1085-93.

17. Ancker JS, Kern LM, Edwards A, Nosal S, Stein DM, Hauser D, Kaushal R. Associations between healthcare quality and use of electronic health record functions in ambulatory care. J Am Med Inform Assoc 2015 Jul;22(4):864-71.

18. Staubert S, Schaaf M, Jahn F, Brandner R, Winter A. Modeling Interoperable Information Systems with $3 \mathrm{LGM}(2)$ and IHE. Methods Inf Med 2015;54(5):398-405.

19. Marceglia S, Fontelo P, Rossi E, Ackerman MJ. A Standards-Based Architecture Proposal for Integrating Patient mHealth Apps to Electronic Health Record Systems. Appl Clin Inform 2015;6(3):488-505.

20. Oral B, Cullen RM, Diaz DL, Hod EA, Kratz A. Downtime procedures for the 21st century: using a fully integrated health record for uninterrupted electronic reporting of laboratory results during laboratory information system downtimes. Am J Clin Pathol 2015 Jan;143(1):100-4.

21. Sax U, Lipprandt M, Röhrig R. The Rising Frequency of IT Blackouts Indicates the Increasing Relevance of IT Emergency Concepts to Ensure Patient Safety. Yearb Med Inform 2016:130-7.
Correspondence to:

Dr. Werner 0 Hackl

Institute of Biomedical Informatics

UMIT - University for Health Sciences, Medical Informatics and Technology

Eduard-Wallnoefer-Zentrum 1

6060 Hall in Tirol, Austria

Tel: + 435086483806

E-mail:werner.hackl@umit.at

Dr. med. Thomas Ganslandt

Medizinisches IK-Zentrum

Universitätsklinikum Erlangen

Glückstr. 11

DE-91054 Erlangen, Germany

Tel +49913185-36712

E-mail: thomas.ganslandt@uk-erlangen.de

\section{Appendix: Content Summa- ries of Selected Best Papers for the IMIA Yearbook 2016 Section "Clinical Information Systems"}

\section{Varpio L, Rashotte J, Day K, King J, Kuziemsky C, Parush A \\ The EHR and building the patient's story: A qualitative investigation of how EHR use obstructs a vital clinical activity}

\section{Int J Med Inform 2015 Dec;84(12):1019-28}

The introduction of electronic health records (EHRs) has in many cases focused on the capture and processing of structured data, enabling knowledge-based functions to support clinical workflows as well as secondary use of acquired data e.g. for research purposes. It should not be overlooked, though, that routine clinical documentation is more than the sum of individual data elements it plays a vital role in the interprofessional coordination of care.

In this paper, Varpio et al. carried out a longitudinal pre/post investigation of an EHR implementation at a tertiary case pediatric hospital, focusing on the impact the EHR had on building the patient's story. The patient's story is described as the "big picture" - an awareness of the patient's current status, relevant history, emergent data patterns during ongoing care, identified problems and the holistic care plan. Building the patient's story was identified as a vital clinical skill essential to provide coordinated interprofessional care and maintain patient safety as well as provider credibility.

After introduction of the EHR platform it was noted that clinical documentation became "splintered" across various workflows, forms and fields. Even though the system allowed the addition of narrative notes to structured forms, they were not displayed in a way that allowed quick assessment. Clinical users described that relevant interconnections between observations were lost and it became difficult to assess the "why" and "how" of team members' actions, obstructing the synthesis of the patient's story. The authors conclude that aspects of fragmentation and the obstruction of interprofessional communication should be considered in the design, selection and adoption of EHR platforms.

\section{Pickering BW, Dong Y, Ahmed A, Giri J, Kilickaya O, Gupta A, Gajic O, Herasevich V}

The implementation of clinician designed, human-centered electronic medical record viewer in the intensive care unit: a pilot step-wedge cluster randomized trial Int J Med Inform 2015 May;84(5):299-307

The display of information in commercially available electronic medical records (EMRs) tends to be structured by data sources (e.g. grouping medications, laboratory or vital signs in separate sections). Especially in data-rich environments like an intensive care unit (ICU), this approach may require multiple accesses to various parts of the record in order to assess relevant data items. In this paper, Pickering et al. describe the evaluation of a locally developed EMR viewer called AWARE (Ambient Warning and Response Evaluation). AWARE accesses relevant data from an underlying EMR system and presents them in a clinician-designed view structured by organ system. The time required for data gathering before morning rounds was measured as a primary outcome measure before and after the introduction of AWARE in a step-wedge trial carried out at 4 consecutive ICUs, accompanied by surveys regarding information management, collaboration aspects and adoption. 
A statistically significant reduction from 12 to 9 minutes required for pre-round data gathering was observed after the introduction of AWARE. Survey results showed a perception of improved information management (timeliness and efficiency, accuracy, clarity of presentation) through AWARE versus the EMR, whereas no significant differences were noted regarding data precision, information content or sufficiency. Aspects of care planning, education, team participation and communication were unchanged pre/ post implementation. In a post implementation survey, $86 \%$ of participants expressed interest in continuing use of AWARE. The authors conclude that the incorporation of practice needs and local development cycles can improve efficiency and usability of data management in EMRs.

\section{Slight SP, Eguale T, Amato MG, Seger AC, Whitney DL, Bates DW, Schiff GD \\ The vulnerabilities of computerized physician order entry systems: a qualitative study}

\section{J Am Med Inform Assoc 2016 Mar;23(2):311-6}

Medication errors are a common problem. While computerized physician order entry (CPOE) has been acknowledged to help prevent such errors, it has also been shown that CPOE systems may not detect relevant errors or actually contribute to erroneous orders. In this article, Slight et al. present an evaluation of 13 commercially available or locally developed CPOE systems in which prescriptions from reported medication errors were systematically entered and reviewed for alerts and usability issues. Prescriptions were extracted out of a random sample of medication error reports from the US Pharmacopeia MEDMARX reporting database, where CPOE was considered a contributing factor.

It was found that the presence, content and severity level of alerts varied widely between the tested systems, and that variations occurred even within the same system at different locations, due to differences in configuration (including inadvertent deactivation of all medication checks) or data entry practices. The wording of alerts was in some cases found to be confusing, as well as the timing (e.g. only after duplicate orders were fully entered and signed off) and content selection (e.g. including warnings for previous prescriptions). The authors conclude that systematic testing of CPOE systems as well as the consideration of human factor principles should be applied to improve the safety of CPOE implementations.

Wright A, McCoy AB, Hickman TT, Hilaire DS, Borbolla D, Bowes WA 3rd, Dixon WG, Dorr DA, Krall M, Malholtra S, Bates DW, Sittig DF

Problem list completeness in electronic health records: A multi-site study and assessment of success factors

Int J Med Inform 2015 0ct;84(10):784-90
Problem lists (PLs) have become an essential component of electronic medical records, contributing not just to the immediate care process, but also to clinical decision support as well as inclusion in registries or individualized treatment programs (e.g. for diabetes management). Despite this, problem lists have previously been shown to be incomplete, inaccurate or out of date. In this paper, Wright et al. present a retrospective analysis of problem list completeness at 10 healthcare organizations for diabetes in comparison to a pathognomonic laboratory measurement, accompanied by interviews at the top performing sites to determine relevant success factors. Surveys were also carried out at all participating sites regarding implementation of those factors.

Problem list completeness varied widely between $60.2 \%$ and $99.4 \%$. Interviews carried out at the 4 top performing sites showed organizational factors (shared responsibility for PL maintenance, an established culture of using the PL), technical factors (gap reporting, link to billing codes, PL-oriented charting) as well as financial factors (PLbased enrollment into special reimbursement programs). A survey across all participating sites showed that implementation of these factors was associated with higher PL completeness. The authors conclude that organizations should consider these practices to improve problem list completeness. 